\title{
Abdomen abierto y cierre temprano de la pared abdominal
}

\author{
Open abdomen and early closure of the abdominal wall
}

\author{
Laura Natalia Ramírez-Méndez¹ $\mathbb{D}$, Neil Valentín Vega-Peña² $\mathbb{D}$, \\ Luis Carlos Domínguez-Torres ${ }^{3}$ (D)
}

1 Médica, residente de Cirugía General, Universidad de La Sabana, Chía, Colombia.

2 MD, MSc, FACS, especialista en Cirugía general, coordinador Departamento de Cirugía, Universidad de La Sabana, Chía, Colombia.

3 MD PhD, especialista en Cirugía general, profesor asociado de Cirugía, Universidad de La Sabana, Chía, Colombia.

\section{Resumen}

El abdomen abierto es una opción terapéutica en pacientes críticamente enfermos. Se utiliza cuando el cierre de la cavidad abdominal no puede o no debe ser realizado. No obstante, su utilidad como parte de una estrategia tradicionalmente aceptada ha disminuido, en la medida en que se han incrementado las secuelas en la pared abdominal, en especial la hernia ventral. Los procedimientos requeridos para la reconstrucción anatómica y funcional de la pared abdominal, como parte del tratamiento de una hernia ventral, revisten una alta complejidad y constituyen un nuevo escenario quirúrgico. Igualmente, conllevan incertidumbre respecto a su naturaleza y posibles complicaciones, además de que condicionan mayores gastos al sistema de salud. Para evitar los problemas del cierre tardío de la pared abdominal, se han desarrollado alternativas para superar el abordaje tradicional de "tratar y esperar", hacia "tratar y reconstruir" tempranamente. El objetivo de la presente revisión es realizar una descripción de los principales avances en el tratamiento del abdomen abierto y el papel del cierre temprano de la pared abdominal, haciendo énfasis en la importancia de un cambio conceptual en el mismo.

Palabras clave: pared abdominal; hernia ventral; hernia incisional; técnicas de abdomen abierto; reconstrucción de pared abdominal; mallas quirúrgicas.

\begin{abstract}
The open abdomen is a therapeutic option in critically ill patients. It is used when the closure of the abdominal cavity cannot or should not be performed. However, its usefulness as part of a traditionally accepted strategy has diminished, as sequelae in the abdominal wall, especially ventral hernia, have increased. The procedures required for the anatomical and functional reconstruction of the abdominal wall, as part of the treatment of a ventral hernia, are highly complex and constitute a new surgical scenario. Likewise, they lead to uncertainty regarding their nature
\end{abstract}

Fecha de recibido: 11/08/2020 - Fecha de aceptación: 02/09/2020 - Fecha de publicación en línea: 06/04/2021

Correspondencia: Neil Valentín Vega-Peña, Universidad de La Sabana, Campus del Puente del Común, Km. 7, Chía, Colombia.

Teléfono: 57 3102189564. Correo electrónico: neil.valentin@unisabana.edu.co

Citar como: Ramírez-Méndez LN, Vega-Peña NV, Domínguez-Torres LC. Abdomen abierto y cierre temprano de la pared abdominal. Rev Colomb Cir. 2021;36:520-30. https://doi.org/10.30944/20117582.749

Este es un artículo de acceso abierto bajo una Licencia Creative Commons - BY-NC-ND https://creativecommons.org/licenses/by-ncnd/4.0/deed.es 
and possible complications, in addition to conditioning higher expenses for the health system. To avoid the problems of delayed closure of the abdominal wall, alternatives have been developed to overcome the traditional "try and wait" approach to "treat and reconstruct" early. The objective of this review is to describe the main advances in the treatment of the open abdomen and the role of early closure of the abdominal wall, emphasizing the importance of a conceptual change in it.

Keywords: abdominal wall; ventral hernia; incisional hernia; open abdomen techniques; abdominal wall reconstruction; surgical mesh.

\section{Introducción}

El abdomen abierto es una opción terapéutica en pacientes críticamente enfermos. Se indica cuando el cierre de la cavidad abdominal no puede ser efectuado (por ejemplo, en presencia de síndrome de hipertensión abdominal), o no debe ser realizado (por isquemia intestinal o peritonitis fecal, entre otros) ${ }^{1}$. Por lo tanto, es un componente del "enfoque inicial de control de daños" utilizado en patologías quirúrgicas abdominales, traumáticas y no traumáticas ${ }^{2-4}$, que alcanza una tasa de mortalidad cercana al $60 \%{ }^{5,6}$.

Como estrategia temporal, el abdomen abierto demanda recursos hospitalarios, tecnológicos, logísticos y científicos, para controlar de forma secuencial una condición que amenaza la vida del paciente. Es una condición que determina una alta estancia hospitalaria e incrementa los costos directos e indirectos de la atención en salud. Sus principales complicaciones son la fístula entero-cutánea, reportada entre el 5,5 \% y el 17,2 $\%$ de los pacientes, las lesiones intestinales y los trastornos hidroelectrolíticos ${ }^{6}$. Una de las secuelas indeseables del abdomen abierto es la hernia ventral, que impacta negativamente la calidad de vida de los pacientes, condiciona una incapacidad laboral prolongada, disminuye la autoestima, y también aumenta los costos en procedimientos diagnósticos y terapéuticos, necesarios para el cierre definitivo de la pared abdominal ${ }^{7-10}$.

Por lo anterior, la evolución del manejo del abdomen abierto ha migrado desde una fase terapéutica inicial de "tratar y esperar" a una nueva de "tratar y reconstruir". En particular, este cambio de paradigma involucra el cierre temprano de la pared abdominal, con el objetivo de disminuir las complicaciones tempranas y las secuelas del abdomen abierto ${ }^{4,11}$. En esta revisión se describen los principales avances en el tratamiento del abdomen abierto y el papel del cierre temprano de la pared abdominal.

\section{Evolución en el tiempo de los dispositivos de recubrimiento abdominal en abdomen abierto}

El manejo del abdomen abierto ha evolucionado desde la primera mitad del siglo XX hasta la actualidad (Figura 1). En 1935 Sperling y Wagensteen lo conceptualizaron como una medida para la prevención de la hipertensión abdominal. Posterior-

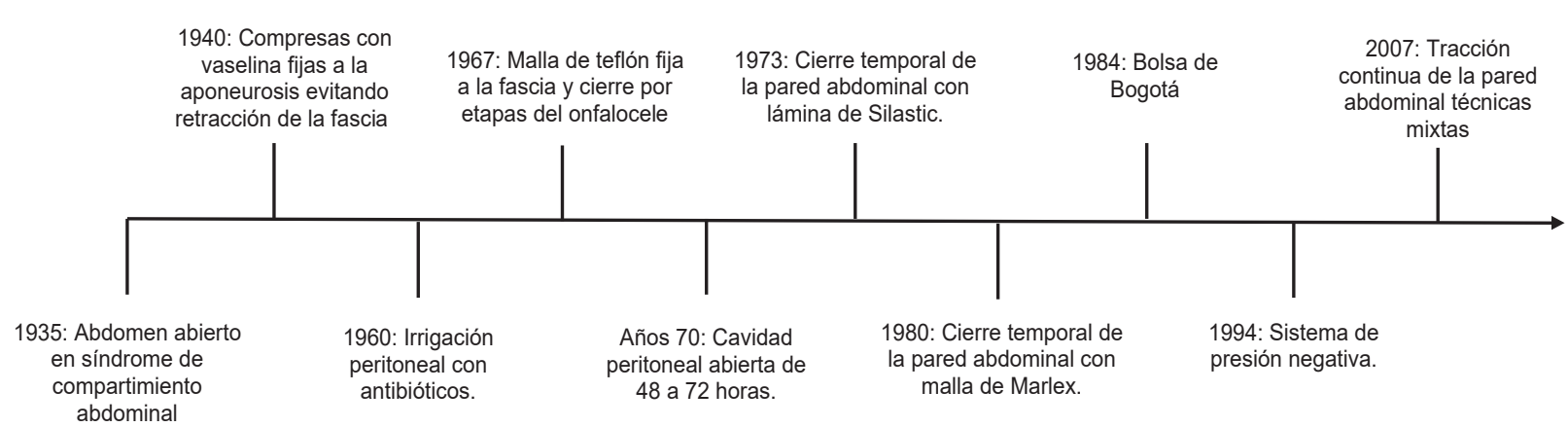

Figura 1. Evolución del abdomen abierto a través de la historia. 
mente, durante la segunda guerra mundial el abdomen abierto se constituyó en una alternativa para el tratamiento de los heridos con lesiones abdominales ${ }^{12}$. En 1940 Olgivie describió el uso de compresas con vaselina fijas a la aponeurosis anterior del abdomen, con el objetivo de evitar su retracción y proteger los órganos intraabdominales ${ }^{12}$. Veinte años después, en 1960 Artz describió la irrigación de la cavidad peritoneal con antibióticos ${ }^{13}$ y Schummer la irrigación continua intraperitoneal ${ }^{14}$.

Los primeros reportes con el uso de dispositivos y mallas fijas a la aponeurosis en el tratamiento del abdomen abierto fueron realizados por Schuster, en 1967, para el cierre por etapas del onfalocele ${ }^{15}$. Igualmente, en los años setenta, Steinberg propuso la reoperacion planeada de la cavidad peritoneal en las primeras 48 a 72 horas ${ }^{16}$. En 1973, Mansberger reportó el cierre temporal de la pared abdominal realizando un cubrimiento de los órganos intraabdominales con una lámina de Silastic ${ }^{17}$ y, en 1980, Teichmann y Wittmann, propusieron el uso de mallas con cremallera para el lavado programado de la cavidad peritoneal ${ }^{18}$. Cuatro años después, Borráez utilizó una lámina de polivinilo para cubrir temporalmente los órganos intraabdominales, la cual fue denominada "Bolsa de Bogotá" 3.

A partir de los años noventa se empezaron a utilizar los sistema de presión negativa en el manejo del abdomen abierto ${ }^{19}$. Finalmente, desde el 2007, se implementó la tracción continua de la pared abdominal, mediante una malla sintética, en combinación con los sistemas de presión negativa, para facilitar el posterior cierre de la pared ${ }^{20}$.

\section{Técnicas para el manejo del abdomen abierto}

Como un recurso terapéutico, el abdomen abierto ha demostrado ser efectivo en disminuir la mortalidad y las complicaciones postoperatorias tempranas, y ha aumentado la supervivencia de los pacientes críticamente enfermos, con patologías traumáticas y no traumáticas de la cavidad abdominal, del $7 \%$ al $65 \%$ en los últimos veinte años ${ }^{21}$. Sin embargo, el tiempo requerido con abdomen abierto depende de las complicaciones que presente el paciente y de la dificultad para el cierre ${ }^{4}$, por lo que es necesario implementar tempranamente el cierre de la pared abdominal, en la medida de lo posible, dentro de los primeros siete días de tratamiento ${ }^{21}$.

Los objetivos actuales del abdomen abierto, aparte de realizar intervenciones especificas en los órganos abdominales, son: 1) evitar la retracción lateral de la aponeurosis de los músculos rectos abdominales; 2) permitir fácilmente las reintervenciones planeadas y no planeadas, y 3) posibilitar un cierre definitivo y temprano de la pared abdominal ${ }^{22}$. Con estos objetivos en mente, también han evolucionado los dispositivos de cierre temporal de la cavidad abdominal. En particular, esta evolución pretende disminuir el uso de sistemas pasivos para el cubrimiento de los órganos de la cavidad abdominal (como la bolsa de Bogotá), e incrementar la utilización de dispositivos dinámicos como los sistemas de presión negativa ${ }^{21,23}$. Por último, en años recientes ha aumentado el uso de técnicas mixtas (figura 2).

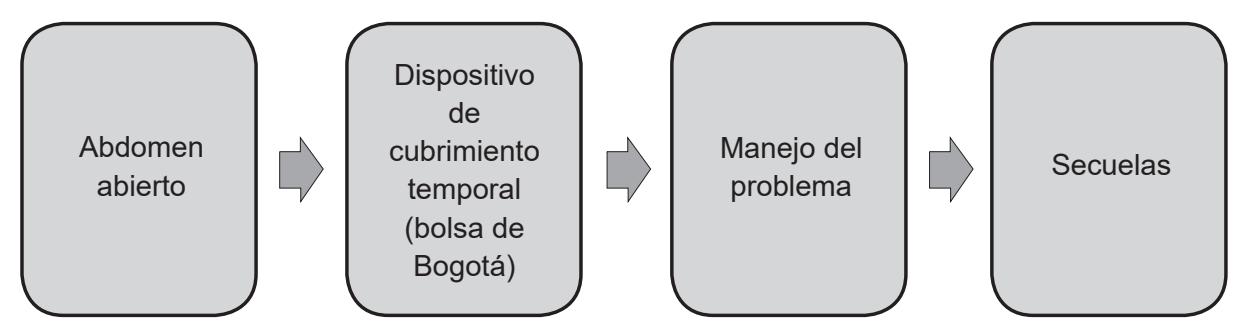

Figura 2. Abordaje tradicional del abdomen abierto. Fuente: Elaboración propia 
Las técnicas para el manejo del abdomen abierto se pueden agrupar en ${ }^{21}$ :

1. Técnicas con cierre de piel: buscan contener los órganos intraabdominales a través del cierre de la piel con suturas, el uso de pinzas de campo quirúrgico (towel clips) y la bolsa de Bogotá ${ }^{21}$. La aparición de fístulas entero-cutáneas ocurre hasta en el 14,4\% de los pacientes sometidos a estas técnicas y el porcentaje de éxito en el cierre primario de la pared abdominal oscila entre el $12 \%$ y el $82 \%$, pero en general, la mayoría de los pacientes evolucionan al cierre tardío de la herida por segunda intención asociado a hernias ventrales ${ }^{21}$.

2. Técnicas con cierre fascial: se basan en la colocación de un material sintético suturado a la vaina aponeurótica de los músculos abdominales para evitar su retracción. Los elementos utilizados para la tracción pueden ser el parche de Wittmann o diferentes tipos de malla de material biológico o sintético. Este medio de tracción utilizado se remueve en el momento del cierre de la pared abdominal ${ }^{21}$. Las reintervenciones se realizan a través de este dispositivo, tratando siempre de aproximar, de forma secuencial y metódica, los bordes músculo-aponeuróticos de la herida en la pared abdominal, con el objetivo de lograr el cierre de la pared abdominal cuando las condiciones clínicas del paciente lo permitan. Se reporta un éxito en el cierre definitivo de hasta el $89 \%$ con el uso de mallas no absorbibles y una incidencia de fístulas entre el $6 \%$ y el $18 \%$ de los pacientes, dependiendo del tipo de dispositivo ${ }^{21}$. Las reintervenciones son realizadas usualmente cada 48 a 72 horas, con una aproximación de la fascia en cada intervención de 2 a $4 \mathrm{~cm}$, por lo que se considera casi que obligatorio el uso de estas técnicas cuando el cierre no se llevará a cabo en la primera semana. De acuerdo con las guías sobre el manejo de la pared abdominal en el abdomen abierto de la Asociación Europea de Hernia (EHS), su utilización como técnica de cierre dinámico debe ser contem- plada (recomendación de carácter fuerte) ${ }^{24}$.

3. Técnicas con sistema de presión negativa: cumplen la función de contener los órganos intraabdominales, prevenir la deshidratación, disminuir el edema visceral, facilitar la aproximación de la pared abdominal y evacuar los fluidos peritoneales ${ }^{25}$. Los estudios reportan éxito en el cierre primario hasta en el $68 \%$ de los pacientes, con una incidencia de fístulas del $5 \%{ }^{26}$. Su utilización se contempla como parte del tratamiento del abdomen abierto, aun cuando un cierre dinámico no pueda ser realizado (recomendación de carácter débil por falta de evidencia), de acuerdo a la EHS ${ }^{24}$.

4. Mixtas: se componen de un método de tracción permanente de los bordes de la fascia mediante mallas sintéticas en forma de "puente" (tracción mediada con malla), asociado a un sistema de presión negativa continúa, como un dispositivo temporal dinámico. Por lo tanto, las técnicas mixtas integran y potencian los beneficios de esas dos intervenciones terapéuticas, estableciendo de forma simultánea un abordaje de la condición intraabdominal subyacente y la pared abdominal. La combinación de sistemas de presión negativa asociado a medios de tracción de fascia ha mostrado tener el porcentaje de éxito más elevado (mayor del $80 \%$ ) en lograr el cierre definitivo de la pared abdominal ${ }^{21}$.

\section{Cierre de la pared abdominal después de abdomen abierto}

Una vez resuelta la condición subyacente al abdomen abierto, el reto siempre debe ser lograr el cierre definitivo de la pared abdominal, de manera temprana, idealmente en la misma hospitalización, con el objetivo de disminuir la mortalidad, complicaciones y estancia hospitalaria ${ }^{4,24}$. El cierre de la pared abdominal debe considerarse según las condiciones fisiológicas, nutricionales e infecciosas del paciente, teniendo presente la distancia entre los bordes de la aponeurosis de los músculos, siendo óptimo un espacio de 3 a $7 \mathrm{~cm}$ entre ellos para lograr ese propósito ${ }^{23,27}$. Cuando estas condiciones no se cumplen, es posible que resulte una 
hernia ventral "planeada", con las implicaciones y complicaciones mencionadas ${ }^{23}$.

La extensión de la utilidad del abdomen abierto hacia las patologías no traumáticas estableció un comportamiento diferencial en el logro del cierre definitivo de la pared abdominal. Aproximadamente, en el $60 \%$ de los pacientes con abdomen abierto secundario a trauma puede lograrse el cierre abdominal definitivo de manera exitosa en la primera semana. Sin embargo, este porcentaje es inferior en los pacientes con condiciones sépticas o inflamatorias abdominales, de origen no traumático, en quienes este cierre definitivo se acerca al $30 \%$ de los $\operatorname{casos}^{23,28}$.

El método ideal para el cierre de la pared abdominal, después del manejo con abdomen abierto, involucra el cierre primario de los músculos y la colocación de una malla de refuerzo ubicada supra (onlay) o infra (sublay) aponeurótica. No obstante, según las características de cada paciente, es posible que se requieran otras alternativas ${ }^{4,24,29}$.

Los estudios acerca de la hernia incisional posterior al cierre de la pared abdominal en pacientes con abdomen abierto son escasos debido a la ausencia de seguimiento rutinario de los pacientes a largo plazo ${ }^{30}$. Sin embargo, se reporta una incidencia del $21 \%$ dentro de los primeros veinte meses de seguimiento y del $54 \%$ en los cinco primeros años ${ }^{31,32}$. Estos porcentajes son superiores frente al $20 \%$ de hernias ventrales después de una laparotomía en condiciones no urgentes ${ }^{10}$.

La literatura hace énfasis en realizar el cierre de la pared abdominal, pero no hay claridad en cuál es la mejor manera para lograrlo. Existen estudios con cierre de la pared abdominal con sutura continua ${ }^{33-35}$, otros argumentan una relación de la longitud de la sutura versus la longitud de la herida quirúrgica de al menos 4:1, o dejan la decisión del uso de sutura continua o interrumpida, así como absorbibles o no absorbibles, a criterio del cirujano ${ }^{34}$. En el momento de elegir la técnica quirúrgica para el cierre de la pared abdominal, pueden adoptarse los postulados de las guías de la EHS para el cierre de la pared abdominal, donde la recomendación en pacientes sin adherencias inter asas, ni a la pared abdominal, sin presencia de fistulas y a quienes se realizara el cierre de la pared abdominal en la misma hospitalización es utilizar sutura continua con monofilamento de reabsorción lenta con una relación longitud de sutura: herida de $4: 1^{24}$, con puntos pequeños en ambos lados de la fascia evitando la tensión en la misma y, contemplando el uso de una malla de refuerzo en casos de alto riesgo de evisceración o eventración ${ }^{10,24,30,36}$.

La técnica de separación de componentes de la pared abdominal, bien sea anterior o posterior, publicada por primera vez por Ramirez y colaboradores ${ }^{37}$, es una opción terapéutica que aumenta las probabilidades de éxito del cierre en un porcentaje superior al $75 \%$ de los casos ${ }^{4}$. En pacientes en que no se logra el cierre del defecto en la pared abdominal, pueden realizarse colgajos del músculo tensor de la fascia lata, de piel o de grupos musculares mediante rotaciones, lo que se considera demandante y requiere de una participación activa por parte de cirugía plástica ${ }^{38}$. Las guías de la EHS no concluyeron con respecto a la realización de estos procedimientos de forma rutinaria previo al cierre de la pared abdominal, dando como sugerencia evaluar detenidamente cada caso y considerando pros y contras de una posible intervención en la pared abdominal, ante la posibilidad de procedimientos quirúrgicos futuros ${ }^{24}$.

La instalación de materiales protésicos entre los músculos (inlay) a manera de "puente" no se aconseja, por la alta tasa de reproducción herniaria, debido al abombamiento progresivo de la pared al no tener un soporte muscular ${ }^{30,39} \mathrm{y}$, por la disfunción a largo plazo de la pared abdominal anterior, que impactan negativamente en la función del tronco (estabilidad, postura, lordosis acentuada), ventilación, estética, autoestima y calidad de vida ${ }^{9,10}$. De forma análoga, la utilización de mallas bioabsorbibles, sintéticas o biológicas, representan una alternativa para el cierre temprano, sin embargo, no se recomiendan por la alta tasa de hernia ventral tardía, por el escaso soporte que brindan a la pared abdominal y por el alto costo ${ }^{30,39,40}$. 
La desinserción de los músculos oblicuos externos puede ser necesaria en defectos de los bordes de la fascia de 10 a $15 \mathrm{~cm}$, y los defectos de mayor tamaño pueden requerir además liberar la hoja posterior del recto ${ }^{23}$. En defectos de gran tamaño se tienen otras opciones como la rotación de los rectos abdominales (rectus turnover flap), donde se libera la vaina anterior del músculo recto y luego se moviliza este colgajo hacia la línea media para realizar el cierre, con o sin material protésico concomitante ${ }^{8}$. Hay reportes del uso de una doble malla complementaria a la separación de componentes ("sandwich"), en los procedimientos reconstructivos, pero no existen estudios que documenten su eficacia en comparación con la instalación de una sola prótesis ${ }^{41,42}$.

Finalmente, el cierre de la pared abdominal con técnicas complejas (separación de componentes o colgajos rotacionales) no se recomienda hasta tanto no esté efectuada la restitución del tracto gastrointestinal (estomas). Especialmente, la alta probabilidad de complicaciones infecciosas locales o la incertidumbre después del cierre del estoma, que pueden implicar deshacer la reconstrucción de la pared abdominal en el caso de complicaciones (fuga anastomótica o reoperación no planeada), advierten sobre la necesidad de utilizar esta técnica con precaución ${ }^{39}$. Si se contempla revertir el estoma de forma futura una vez el paciente este recuperado y se considere un cierre de la pared abdominal de forma simultánea, este podría efectuarse adicionando a la eventual separación de componentes de la pared, una prótesis en posición supra aponeurótica, preferiblemente macro porosa, que posibilite el control de una eventual infección del sitio operatorio superficial o profundo, sin deshacer el procedimiento reconstructivo y el retiro de la prótesis ${ }^{30}$.

\section{Cierre temprano de la pared abdominal en abdomen abierto: un objetivo a considerar}

El cierre temprano de la pared abdominal en abdomen abierto, definido como aquel que se realiza durante el tratamiento inicial del paciente, en la misma hospitalización ${ }^{43}$, es un concepto que ha venido evolucionando y tomando más fuerza en los últimos años. Este tipo de reconstrucción busca ofrecer mejores resultados a los pacientes, gracias a las diferentes opciones terapéuticas disponibles, tanto para el manejo del abdomen abierto como para el cierre definitivo de la pared abdominal ${ }^{30}$. La utilización de técnicas mixtas durante el manejo del abdomen abierto, han impactado de manera positiva el pronóstico de los pacientes y el logro del cierre definitivo de la pared abdominal ${ }^{21}$. Con la tecnología a favor del paciente y el cirujano, un equipo quirúrgico coordinado y una estrategia dirigida a cada paciente y su condición particular, se puede lograr un cierre definitivo y temprano de la pared abdominal en un paciente con abdomen abierto. En la figura 3 se ejemplifica la conceptualización actual en el cierre de la pared en pacientes con abdomen abierto.

Debido a la complejidad de manejo de los pacientes, en enero de 2009 se publicó un consenso para clasificar el abdomen abierto, buscando describir el curso clínico, estandarizar el manejo y comparar los pacientes por características

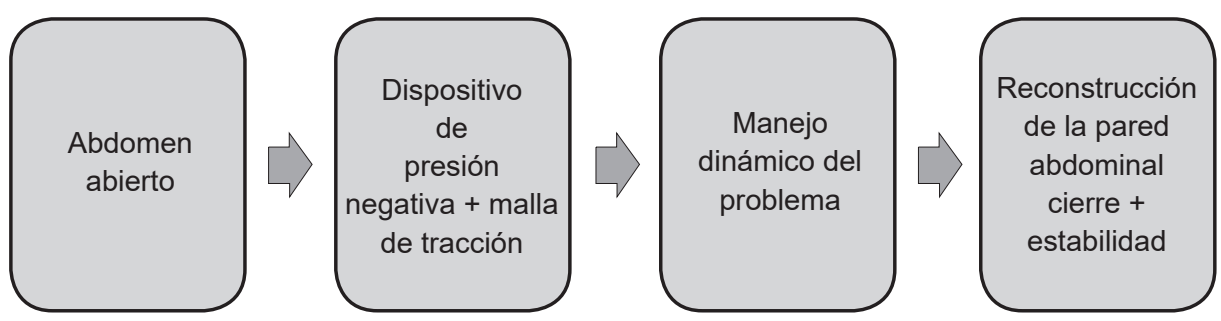

Figura 3. Conceptualización del abdomen abierto y la reconstrucción de la pared como manejo integrado. Fuente: elaboración por los autores. 
similares, con el fin de optimizar el cierre de la pared abdominal ${ }^{38}$. Los resultados del consenso concluyeron que en los pacientes con abdomen abierto grado I(A/B) - II(B) donde el abdomen aún no está congelado y no hay presencia de fístulas, debe procurarse el control de la infección en caso de su persistencia y evitar la fijación de las asas a la pared abdominal, para llevar a cabo un cierre temprano de la pared abdominal. En este punto específico, las guías de EHS consideran como recomendación fuerte el uso de técnicas de cierre dinámico en este grupo de pacientes ${ }^{24}$.

En el manejo del abdomen abierto deben generarse estrategias para evitar un grado III - IV del abdomen abierto, donde hay presencia de fístulas entero-cutáneas o un abdomen "congelado", y la prioridad del manejo deja de ser el cierre de la pared abdominal ${ }^{38}$.

Se propone un manejo basado en una política de "tratar y reconstruir". Al momento de la laparotomía inicial, considerando la conducta de un abdomen abierto, es función del cirujano evaluar todas las condiciones y definir una estrategia de cierre de la pared abdominal en este momento o aceptar una hernia ventral "planeada". En caso de elegir el cierre temprano, debe establecerse un plan terapéutico para lograr el objetivo, idealmente en la primera semana. Para ello, se efectúan procesos de toma de decisiones en términos del mecanismo de cierre temporal de la pared a utilizar, del momento ideal para el inicio de un sistema de presión negativa, y de los métodos de tracción fascial. Teniendo en cuenta el estado crítico de estos pacientes, los cuidados postoperatorios se realizan en la unidad de cuidados intensivos, donde es requerido un trabajo en equipo junto con el grupo quirúrgico. Nuevas laparotomías son requeridas en el manejo del paciente hasta lograr condiciones óptimas para el cierre de la pared abdominal ${ }^{11,30}$.

De acuerdo con un estudio de cohorte efectuado en 87 pacientes sépticos, y tras una técnica mixta (terapia de presión negativa + tracción fascial con malla ), fue posible un cierre definitivo de la pared abdominal en el $78 \%$ de los pacientes, tras 12,6 días y 4,3 reoperaciones ${ }^{35}$. En caso de no lograrse, se debe aceptar una hernia ventral "planeada", con una expectativa de reconstrucción de la pared abdominal en los siguientes 6 a 12 meses $^{11,39,44}$.

La prevención de una hernia incisional a largo plazo, en este tipo de pacientes en los cuales se ha logrado un cierre de la pared abdominal de forma primaria tras un abdomen abierto, es un tema poco estudiado en la actualidad. La utilización de una malla profiláctica como refuerzo aponeurótico termina siendo la mejor recomendación, teniendo en cuenta que la suma de todas las condiciones del paciente condicionan un tejido musculo-aponeurótico de inferior calidad, frágil, traumatizado por las cirugías previas y en un estado metabólico de catabolismo activo propio de la fase en que se encuentra el paciente. Todo esto, condiciona un riesgo mayor de hernia incisional y lo que pareciera ser un cierre primario de la pared abdominal con una adecuada técnica, parece no ser suficiente para establecer una matriz de tejido cicatricial fuerte y duradero ${ }^{24,30}$.

El principal temor en la instalación de una malla de refuerzo al momento del cierre de la pared abdominal es la infección del sitio operatorio que se incrementa hasta en un $30 \%{ }^{30}$. No obstante, al evaluarse el riesgo/beneficio de su utilidad como medida profiláctica ante una hernia incisional, se soporta su implementación rutinaria, a pesar de las consideraciones infecciosas antes anotadas. Bajo esta perspectiva, la posición supramuscular (onlay) constituye una ventaja con respecto a la facilidad de instalación y el control de un eventual proceso infeccioso futuro ${ }^{24,30,45}$. Existe un vacío en el conocimiento en este punto específico, estableciéndose una oportunidad de realizar investigaciones en el tema.

\section{Consideracionesparalaimplementación de una estrategia de cierre temprano de la pared abdominal}

Es necesario generar conciencia en los cirujanos acerca de la importancia de contemplar, de forma simultánea, el manejo del abdomen abierto y el cierre temprano de la pared abdominal. La meta es evitar que un método de recubrimiento de la pared abdominal temporal se convierta en un ma- 
nejo definitivo, con las secuelas mencionadas. Hoy en día existen, con mayor frecuencia, opciones terapéuticas para lograr el cierre temprano de la pared abdominal, pero aun así, no siempre están disponibles, o se restringen a los centros médicos con mayores recursos. Los obstáculos administrativos y limitaciones económicas no pueden ser los determinantes en el manejo final de los pacientes con abdomen abierto.

Es recomendable la creación e implementación de protocolos para el manejo del abdomen abierto, que considere esta estrategia integradora. Igualmente, procurar un trabajo en equipo al interior de los grupos quirúrgicos, donde todos sus integrantes conozcan las técnicas e insumos, así como una comunicación fluida ante las eventualidades en el proceso. El desconocimiento de las técnicas quirúrgicas no debe ser una limitante para su implementación, teniendo en cuenta las bondades del procedimiento.

Es necesaria una mejora de las competencias técnicas y del conocimiento de las opciones terapéuticas y avances tecnológicos en los casos de abdomen abierto, por parte del cirujano y el equipo quirúrgico, buscando evitar el impacto negativo para el paciente, las instituciones, los aseguradores y el cirujano, para quien la hernia ventral "planeada" se convierte en un verdadero reto quirúrgico (tabla 1 ).

\section{Comentarios finales}

El manejo del abdomen abierto y el cierre temprano de la pared abdominal es una estrategia terapéutica en evolución. Su entendimiento e implementación como un proceso terapéutico que impacta favorablemente al paciente y todos los involucrados en su manejo, es un imperativo de las instituciones donde se traten pacientes de esta complejidad. La condición crítica de los pacientes con abdomen abierto y la incertidumbre en las expectativas de los tratamientos, obligan a un trabajo consensuado y en equipo, procurando establecer de forma simultánea, en la medida de lo posible, objetivos terapéuticos al interior del abdomen, en la pared abdominal y en los diferentes sistemas orgánicos comprometidos. Existen alternativas para el manejo de estos pacientes, que deben ser evaluadas por el cirujano y el grupo quirúrgico tratante, dentro de una aproximación individualizada para cada caso, en pos de procurar un cierre temprano de la pared abdominal como un propósito fundamental. Evitar la hernia ventral "planeada" trae resultados favorables tanto para el paciente, el cirujano, la institución y el sistema de salud.

Tabla 1. Consideraciones para la implementación del cierre temprano del abdomen

\begin{tabular}{|c|c|c|}
\hline & Favorece el cierre temprano & Limita el cierre temprano \\
\hline $\begin{array}{l}\text { Factores } \\
\text { relacionados con } \\
\text { el paciente }\end{array}$ & $\begin{array}{l}\text {-Prevención de una hernia ventral tardía y } \\
\text { secuelas } \\
\text {-Evitar los riesgos asociados con nuevas } \\
\text { cirugías } \\
\text {-Impacto positivo en la calidad de vida } \\
\text { - Menor incapacidad laboral }\end{array}$ & $\begin{array}{l}\text {-Estado crítico. } \\
\text {-Comorbilidades: anticoagulación, ostomía, } \\
\text { aneurisma aorta abdominal, fístula entero-cutánea, } \\
\text { proceso infeccioso asociado no controlado. } \\
\text { - Necesidad de reintervenciones repetidas posterior } \\
\text { a las primeras dos semanas de manejo de su } \\
\text { abdomen abierto }\end{array}$ \\
\hline $\begin{array}{l}\text { Factores } \\
\text { relacionados con } \\
\text { el cirujano }\end{array}$ & $\begin{array}{l}\text {-Evitar reconstrucciones tardías técnicamente } \\
\text { complejas. } \\
\text {-Variedad de técnicas quirúrgicas. }\end{array}$ & $\begin{array}{l}\text { - Desconocimiento de la técnica quirúrgica y el } \\
\text { concepto integrador. } \\
\text {-Ausencia de trabajo en equipo al interior de la } \\
\text { institución. }\end{array}$ \\
\hline $\begin{array}{l}\text { Factores } \\
\text { relacionados con } \\
\text { la institución }\end{array}$ & $\begin{array}{l}\text {-Disminución de estancia hospitalaria. } \\
\text {-Egreso hospitalario temprano. } \\
\text { - Menores costos directos e indirectos de la } \\
\text { atención en salud. } \\
\text {-Política de mejoramiento de la calidad de } \\
\text { la institución. }\end{array}$ & $\begin{array}{l}\text {-Restricciones administrativas. } \\
\text {-No hay disponibilidad de protocolos. } \\
\text {-No hay disponibilidad de insumos. }\end{array}$ \\
\hline
\end{tabular}




\section{Cumplimiento de normas éticas}

Consentimiento informado: Este estudio es una revisión de la literatura, y como tal no hay necesidad de un consentimiento informado ni de aprobación del Comité de Ética Institucional.

Conflicto de interés: Ninguno declarado por los autores.

Fuente de financiación: La ejecución del escrito responde a un interés académico y no ha sido soportada económicamente por alguna fuente externa.

Contribuciones de los autores: Los autores manifestamos haber participado de forma integral en las diferentes fases del trabajo, tales como: Concepción y diseño del estudio, adquisición de datos, análisis e interpretación de la información, redacción y revisión crítica del manuscrito. Adicionalmente, contribuimos en la elaboración de las gráficas.

\section{Referencias}

1. Schein M. Surgical management of intra-abdominal infection: Is there any evidence? Langenbecks Arch Surg. 2002;387:1-7. https://doi.org/10.1007/s00423-002-0276-Z

2. Chen Y, Ye J, Song W, Chen J, Yuan Y, Ren J. Comparison of outcomes between early fascial closure and delayed abdominal closure in patients with open abdomen: A systematic review and meta-analysis. Gastroenterol Res Pract. 2014;2014:1-8. https://doi.org/10.1155/2014/784056

3. Borráez OA. Abdomen abierto: la herida más desafiante. Rev Colomb Cir. 2008;23:204-9.

4. Coccolini F, Roberts D, Ansaloni L, Ivatury R, Gamberini $\mathrm{E}$, Kluger $\mathrm{Y}$, et al. The open abdomen in trauma and non-trauma patients: WSES guidelines. World J Emerg Surg. 2018;13:1-16. https://doi.org/10.1186/s13017-018-0167-4

5. Ozguc H, Paksoy E, Ozturk E. Temporary abdominal closure with the vacuum pack technique: A 5-year experience. Acta Chir Belg. 2008;108:414-9. https://doi.org/10.1080/00015458.2008.11680252

6. Atema JJ, Gans SL, Boermeester MA. Systematic review and meta-analysis of the open abdomen and temporary abdominal closure techniques in non-trauma patients. World J Surg. 2015;39:912-25. https://doi.org/10.1007/s00268-014-2883-6

7. Arai M, Kim S, Ishii H, Hagiwara J, Kushimoto S, Yokota $\mathrm{H}$. The long-term outcomes of early abdominal wall reconstruction by bilateral anterior rectus abdominis sheath turnover flap method in critically ill patients requiring open abdomen. World J Emerg Surg. 2018;13:1-6. https://doi.org/10.1186/s13017-018-0200-7
8. Arai M, Kushimoto S, Kim S, Masuno T, Hagiwara J, Ishii $\mathrm{H}$, Yokota $\mathrm{H}$. A novel technique for managing open abdomen with the combined use of mesh-mediated traction and the bilateral anterior rectus abdominis sheath turnover flap method: how to do it. Surg Today. 2015;45:1335-9. https://doi.org/10.1007/s00595-015-1133-3

9. Jensen KK, Munim K, Kjaer M, Jorgensen LN. Abdominal wall reconstruction for incisional hernia optimizes truncal function and quality of life: A prospective controlled study. Ann Surg. 2017;265:1235-40. https://doi.org/10.1097/SLA.0000000000001827

10. Muysoms FE, Antoniou SA, Bury K, Campanelli G, Conze J, Cuccurullo D, et al. European Hernia Society guidelines on the closure of abdominal wall incisions. Hernia. 2015;19:1-24.

https://doi.org/10.1007/s10029-014-1342-5

11. Sharrock AE, Barker T, Yuen HM, Rickard R, Tai N. Management and closure of the open abdomen after damage control laparotomy for trauma. A systematic review and meta-analysis. Injury. 2016;47:296-306. https://doi.org/10.1016/j.injury.2015.09.008

12. Iñaguazo S. D, Astudilllo A. MJ. Abdomen abierto en la sepsis intraabdominal severa. ¿Una indicación beneficiosa? Rev Chil Cir. 2009;61:294-300.

https://doi.org/10.4067/S0718-40262009000300014

13. Artz CP, Barnett WO, Crogan JB. Further studies concerning the pathogenesis and treatment of peritonitis. Ann Surg. 1962;155:756-67. https://doi.org/10.1097/00000658-19620500000015

14. Schumer W, Lee DK, Jones B. Peritoneal lavage in postoperative therapy of late peritoneal sepsis preliminary report. Surgery. 1964;55:841-5.

15. Schuster SR. A new method for staged repair of large omphaloceles. Surg Gynecol Obs. 1967;125:837-50.

16. Steinberg D. On leaving the peritoneal cavity open in acute generalized suppurative peritonitis. Am J Surg. 1979;137:216-20. https://doi.org/10.1016/0002-9610(79)90148-X

17. Mansberger AR, Kang JS, Beebe HG, Le Flore I. Repair of massive acute abdominal wall defects. J Trauma Acute Care Surg. 1973;13:766-74.

https://doi.org/10.1097/00005373-197309000-00002

18. Teichmann W, Wittmann DH, Andreone PA. Scheduled reoperations (etappenlavage) for diffuse peritonitis. Arch Surg. 1986;121:147-52.

https://doi.org/10.1001/archsurg.1986.01400020033002

19. Morykwas MJ, Argenta LC, Shelton-Brown EI, McGuirt WBS. Vacuum-assisted closure: a new method for wound control and treatment. Animal studies and basic foundation. Ann Plast Surg. 1997;38:553-62. https://doi.org/10.1097/00000637-199706000-00001 
20. Acosta S, Björck M, Petersson U. Vacuum-assisted wound closure and mesh-mediated fascial traction for open abdomen therapy - A systematic review. Anaesthesiol Intensive Ther. 2017;49:139-45. https://doi.org/10.5603/AIT.a2017.0023

21. Regner JL, Kobayashi L, Coimbra R. Surgical strategies for management of the open abdomen. World J Surg. 2012;36:497-510. https://doi.org/10.1007/s00268-011-1203-7

22. Poortmans N, Berrevoet F. Dynamic closure techniques for treatment of an open abdomen: an update. Hernia. 2020;24:325-31.

https://doi.org/10.1007/s10029-020-02130-9

23. Carnicer-Escusol E. El cierre temporal de la cavidad abdominal: una revisión. Rev Hispanoam Hernia. 2015;3:49-58. https://doi.org/10.1016/j.rehah.2015.02.005

24. López-Cano M, García-Alamino JM, Antoniou SA, Bennet D, Dietz UA, Ferreira F, et al. EHS clinical guidelines on the management of the abdominal wall in the context of the open or burst abdomen. Hernia. 2018;22:921-39. https://doi.org/10.1007/s10029-018-1818-9

25. Barker DE, Kaufman HJ, Smith LA, Ciraulo DL, Richart CL, Burns RP. Vacuum pack technique of temporary abdominal closure: A 7-year experience with 112 patients. J Trauma. 2000;48:201-7. https://doi.org/10.1097/00005373-200002000-00001

26. Barker DE, Green JM, Maxwell RA, Smith PW, Mejia VA, Dart BW, et al. Experience with vacuum-pack temporary abdominal wound closure in 258 trauma and general and vascular surgical patients. J Am Coll Surg. 2007;204:784-92. https://doi.org/10.1016/j.jamcollsurg.2006.12.039

27. Acosta S, Bjarnason T, Petersson U, Pålsson B, Wanhainen A, Svensson M, et al. Multicentre prospective study of fascial closure rate after open abdomen with vacuum and mesh-mediated fascial traction. Br J Surg. 2011;98:735-43. https://doi.org/10.1002/bjs.7383

28. Cristaudo AT, Jennings SB, Hitos K, Gunnarsson R, DeCosta A. Treatments and other prognostic factors in the management of the open abdomen: A systematic review. J Trauma Acute Care Surg. 2017;82:407-18. https://doi.org/10.1097/TA.0000000000001314

29. Kääriäinen M, Kuokkanen H. Primary closure of the abdominal wall after "open abdomen" situation. Scand J Surg. 2013;102:20-4. https://doi.org/10.1177/145749691310200105

30. Berrevoet F. Prevention of incisional hernias after open abdomen treatment. Front Surg. 2018;5:1-5. https://doi.org/10.3389/fsurg.2018.00011

31. Bjørsum-Meyer T, Skarbye M, Højsgaard Jensen K. Vacuum with mesh is a feasible temporary closure device after fascial dehiscence. Dan Med J. 2013;60:A4719.
32. Willms A, Güsgen C, Schaaf S, Bieler D, von Websky M, Schwab R. Management of the open abdomen using vacuum-assisted wound closure and mesh-mediated fascial traction. Langenbeck's Arch Surg. 2014;400:91-9. https://doi.org/10.1007/s00423-014-1240-4

33. Sörelius K, Wanhainen A, Acosta S, Svensson M, Djavani-Gidlund K, Björck M. Open abdomen treatment after aortic aneurysm repair with vacuum-assisted wound closure and mesh-mediated fascial traction. Eur J Vasc Endovasc Surg. 2013;45:588-94. https://doi.org/10.1016/j.ejvs.2013.01.041

34. Brandl A, Laimer E, Perathoner A, Zitt M, Pratschke J, Kafka-Ritsch R. Incisional hernia rate after open abdomen treatment with negative pressure and delayed primary fascia closure. Hernia. 2014;18:105-11. https://doi.org/10.1007/s10029-013-1064-0

35. Fortelny RH, Hofmann A, Gruber-Blum S, Petter-Puchner AH, Glaser KS. Delayed closure of open abdomen in septic patients is facilitated by combined negative pressure wound therapy and dynamic fascial suture. Surg Endosc. 2014;28:735-40.

https://doi.org/10.1007/s00464-013-3251-6

36. Israelsson LA, Millbourn D. Prevention of incisional hernias: How to close a midline incision. Surg Clin North Am. 2013;93:1027-40. https://doi.org/10.1016/j.suc.2013.06.009

37. Ramirez OM, Ruas E, Dellon AL. "Components separation" method for closure of abdominal-wall defects: An anatomic and clinical study. Plast Reconstr Surg. 1990;86:519-26. https://doi.org/10.1097/00006534-199009000-00023

38. Björck M, Bruhin A, Cheatham M, Hinck D, Kaplan M, Manca G, et al. Classification-important step to improve management of patients with an open abdomen. World J Surg. 2009;33:1154-7. https://doi.org/10.1007/s00268-009-9996-3

39. Petro CC, Rosen MJ. Fight or flight: The role of staged approaches to complex abdominal wall reconstruction. Plast Reconstr Surg. 2018;142:38S-44S. https://doi.org/10.1097/PRS.0000000000004847

40. López-Cano M, Brandsma HT, Bury K, Hansson B, Kyle-Leinhase I, Alamino JG, et al. Prophylactic mesh to prevent parastomal hernia after end colostomy: a meta-analysis and trial sequential analysis. Hernia. 2017;21:177-89. https://doi.org/10.1007/s10029-016-1563-x

41. Jernigan TW, Fabian TC, Croce MA, Moore N, Pritchard FE, Minard G, Bee T. Staged management of giant abdominal wall defects: Acute and long-term results. Ann Surg. 2003;238:349-57.

https://doi.org/10.1097/01.sla.0000086544.42647.84 
42. Bröker M, Verdaasdonk E, Karsten T. Components separation technique combined with a double-mesh repair for large midline incisional hernia repair. World J Surg. 2011;35:2399-402. https://doi.org/10.1007/s00268-011-1249-6

43. Scott BG, Welsh FJ, Pham HQ, Carrick MM, Liscum KR, Granchi TS, et al. Early aggressive closure of the open abdomen. J Trauma. 2006;60:17-22.

https://doi.org/10.1097/01.ta.0000200861.96568.bb

44. Dutton WD, Diaz JJ, Miller RS. Critical care issues in managing complex open abdominal wound. J Intensive Care Med. 2012;27:161-71.

https://doi.org/10.1177/0885066610396162
45. Jairam AP, Timmermans L, Eker HH, Pierik RE, van Klaveren D, Steyerberg EW, et al. Prevention of incisional hernia with prophylactic onlay and sublay mesh reinforcement versus primary suture only in midline laparotomies (PRIMA): 2-year follow-up of a multicentre, double-blind, randomised controlled trial. Lancet. 2017;390:567-76.

https://doi.org/10.1016/S0140-6736(17)31332-6 